\title{
A NILM method for cooling load disaggregation based on artificial neural network
}

\author{
Ziwei Xiao, Jiaqi Yuan, Wenjie Gang*, Chong Zhang, and Xinhua Xu
}

Department of Building Environment and Energy Application Engineering, Huazhong University of Science and Technology, China

\begin{abstract}
The demand of building energy management has increased due to high energy saving potentials. Load monitor and disaggregation can provide useful information for building energy management systems with detailed and individual loads of the building, so corresponding energy efficient measures can be taken to reduce the energy consumption of buildings. The technique is investigated widely in residential buildings known as Non-Intrusive Load Monitoring (NILM). However, relevant studies are not sufficient for nonresidential buildings, especially for the cooling loads. This paper proposes a NILM method for cooling load disaggregation using artificial neural network. The cooling load is disaggregated into four categories: building envelope load, occupant load, equipment load and fresh air load. Two approaches are used to realize the load disaggregation: one is based on the Fourier transfer of the cooling loads, the other takes the cooling load, dry-bulb temperature and humidity of outdoor air, and time as inputs. By implementing the methods in a metro station, the performance of the proposed method can be obtained. Results show that both approaches can realize the load disaggregation accurately, with a RMSE less than 11.2. The second approach is recommended with a higher accuracy.
\end{abstract}

\section{Introduction}

Buildings can account for a high percent of the energy consumption of the society. Efficient management of building energy systems can reduce the energy consumption of buildings significantly. Accurate load monitors and prediction of the total cooling and heating loads can help optimize the control and operation of heating, ventilation and air conditioning systems (HVACs), which is widely investigated. Actually, it is more effective to obtain specific energy consumption statistics so that users can devise load-scheduling strategies for optimal energy utilization. However, usually only the total cooling load or heating load can be monitored in practical projects, the detailed load such as the load resulting from the building envelope, the occupants or the fresh air is usually not monitored. It would be very helpful if each individual load can be disaggregated from the monitored total cooling or heating load, which is called load disaggregation in this paper.

The load disaggregation can be realized in two ways. One way is to install the monitor system for each component of the cooling load, which is called intrusive load monitoring. This monitor method can be accurate but the disadvantage is also very apparent which would incur high hardware cost, sensor configuration and installation complexity[1]. Therefore, the non-intrusive load monitoring (NILM) is often preferred. The NILM technique employs intelligent algorithms to disaggregate the total energy consumption collected by a smart meter into individual appliances and identify the loads without adding intrusive sensors [2]. It is taken as a high tech viable solution to achieve an improved demand side energy usage by contributing energy consumption feedbacks and progressive diagnosis mechanisms[3]. A review of appliance-specific feedback literature shows that providing such information can reduce household energy consumption by $14 \%$ on average[4]. Many studies have been conducted in NILM. Kaustav Basu et al.[5] proposed an industrial scale solution which uses a specific modeling technique for appliance detection, trained and tested on two databases from actual customers readings. D. Srinivasan et al. [6] proposes a neural-network (NN)based approach to nonintrusive harmonic source identification. A hybrid, computationally efficient, algorithm for NILM, called Hybrid Signature-based Iterative Disaggregation (HSID), based on the combination of Factorial Hidden Markov Models is proposed for residential power load disaggregation. The method is robust to signal noise and high number of appliances, and can be used in semi-supervised applications.

Currently the studies are mainly about the electricity disaggregation for residential buildings with different appliances. The appliance often has only on/off state or discrete load, which is easy to disaggregate using supervised or non-supervised method. However, the thermal load disaggregation for non-residential buildings is also important to optimize the HVAC operation by providing sufficient feedback information. Relevant studies are not sufficient yet. The main challenge is that 
the load consisting of the thermal load of buildings is usually continuous rather than on/off state.

In China, the subway system is developing rapidly in recent decade and it now comprises $73.4 \%$ of urban railway transit[7]. The HVAC system can contribute to $35 \%$ of total energy consumption so it is necessary to improve the energy efficiency of metro stations. The NILM can be an effective alternative. However, relevant studies are not found yet.

To fill the above research gap, a cooling load NILM method is proposed in this study to disaggregate the cooling load into different categories based on artificial neural networks (ANNs). Two approaches are used, one is based on the transformation of monitored cooling load, the other is based on the load and outdoor weather. A typical metro station located in hot summer and cold winter area of China is selected to demonstrate the effectiveness of this method.

This paper attempts to disaggregate the cooling load based on artificial neuron network. The organization of this paper is as follows: Section 2 introduces the method to realize the load disaggregation. A metro station is introduced in Section 3 as a case study. Results and discussions are presented in Section 4, followed by the conclusions in Section 5.

\section{Method to realize the cooling load disaggregation}

The method used in this paper is shown in Fig . 1 . Detailed steps are explained as follows.

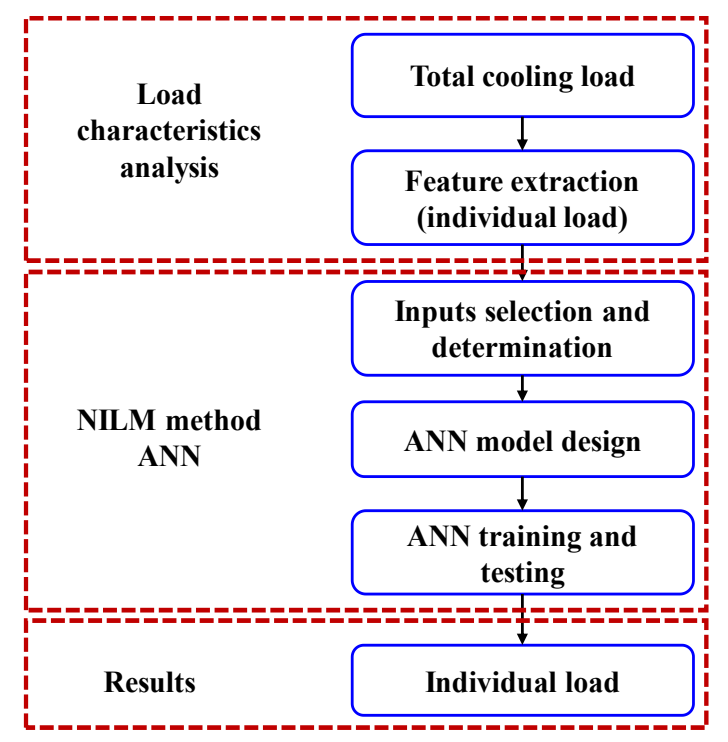

Fig. 1. Steps for cooling load disaggregation

\subsection{Load analysis and characteristics}

The cooling loads of buildings result from occupants, penetrating wind, fresh air, building envelope, indoor equipment and lighting. Different heat gains sources can lead to very different load profiles. The metro station is very different from the general office buildings or public buildings because they usually locate underground. Based on the load source and characteristics, the total cooling load for the metro station can be separated into four types: the load from building envelope, the load from fresh air (including mechanical air supply systems and infiltration trough the entrances), the load from occupants (passengers getting on and off the train) and the load from the equipment (lighting, elevator, LED screen, etc.). Each type of load has special characteristics.

The occupant load is determined by the schedule and density of occupants. The building envelope of metro station is determined by the temperature of underground soil. The fresh air and infiltration loads are determined by the outdoor air temperature and humidity. The equipment load is determined by the lighting, elevator, lifts and other equipment, which almost remains the same throughout the day. All partial loads are normalized to between 0 and 1 so that their characteristics can be clearly seen. The load profiles of these four loads are very different as shown in Fig. 2.

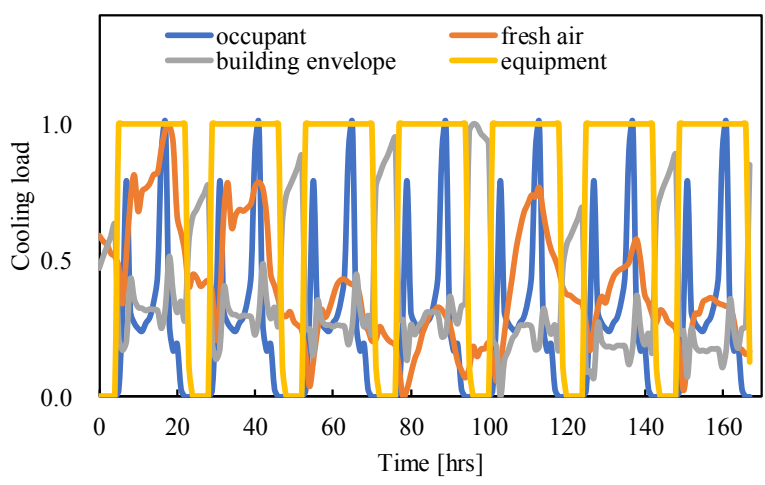

Fig. 2. Profiles of four loads in a typical week

\subsection{NILM based on ANN}

In this study, ANN is used to realize the load disaggregation. ANN has been widely used and investigated since the 1980s[8]. It mimics the human brain neurons in the form of information processing. A neural network is an operational model consisting of a large number of neurons connected to each other. Each node represents a specific output function called an activation function. The connection between every two node represents a weighting value of passing the connection signal, called weight, which is equivalent to the memory of the artificial neural network. The output of the network varies depending on the connection method of the network, the weight value and the excitation function.

In this study, the three-layer back propagation (BP) networks model is used for cooling load NILM. The ANN model is shown in figure 3 . The input layer receives signals and data from the external. The output layer exports the output of the system processing result. The hidden layer learns the connection weights between neurons, namely the connection strength between units, while the representation and processing of information are embodied in the connection relationships of network processing units. 


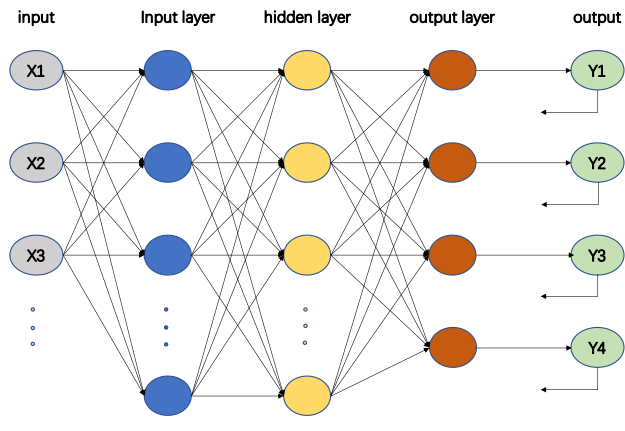

Fig. 3. Typical ANN model

The input selection of ANN models is important to the disaggregation accuracy. Two approaches are used to realize the cooling load disaggregation. One is to transfer the hourly cooling load of a day into a Fourier series consisting of sines and cosines with different frequencies. The coefficients of waves are used as the inputs of the ANN model. The second approach is to use the cooling load directly as the input, together with the outdoor drybulb temperature, relative humidity and time. Since these four kinds of loads have certain periodicity, the analysis will become easier after Fourier transform is applied to transform them from spatial domain to frequency domain. The essence of Fourier transforms is to separate a signal into the addition of an infinite number of sinusoidal signals, while the essence of load decomposition is the separation of signals. When Fourier coefficient and time are taken as inputs, neurons are allowed to learn the weight combination of four kinds of loads at different times to achieve the purpose of decomposition. In addition, because the heat transfer of building envelope and fresh air load are greatly related to outdoor parameters, while the human flow is related to time. Therefore, the second input can be selected. When the inputs are determined, the model can be designed. The number of the neurons in the hidden lay is based on the rule of $2 * n+1$ ( $n$ is the number of inputs) according to Kolmogorov theorem. The learning algorithm is using a feedforward network of the Back Propagation learning algorithm. The neuron transformation function of the BP network uses a sigmoid function (Sigmoid function), so the output is a continuous quantity between 0 and 1 , which can realize arbitrary nonlinear mapping from input to output. The samples of network training and testing are selected randomly. $75 \%$ of samples are used for training and the left are used for testing.

\subsection{Result analysis and assessment criteria}

To evaluate the quality of disaggregation, the commonly used root mean square error (RMSE), and the coefficient of determination (R2) is selected as evaluation indices (Eq. 1 2). Where, $\mathrm{N}$ is the number of samples, $\mathrm{yi}$ is the real value and $\widehat{y}_{i}$ is the outputs from the ANN model.

$$
\begin{aligned}
& \mathrm{RMSE}=\sqrt{\left(\frac{1}{\mathrm{~N}} \sum_{\mathrm{i}=1}^{\mathrm{N}}\left(\widehat{\mathrm{y}}_{\mathrm{i}}-\mathrm{y}_{\mathrm{i}}\right)^{2}\right.} \\
& R^{2}=1-\frac{\sum_{i=1}^{N}\left(y_{i}-\widehat{y_{l}}\right)^{2}}{\sum_{i=1}^{N}\left(y_{i}-\overline{y_{l}}\right)^{2}}
\end{aligned}
$$

\section{Case study for a metro station}

In this research, the method is implemented in a metro station in Wuhan, which locates in the hot summer and cold winter area of China. The cooling load of the metro station is monitored and disaggregated. The metro station has two floors underground with a total area around 3900 $\mathrm{m} 2$. It has four entrances and exits. The operation time of the metro station is from 6 am to 10:30 am every day.

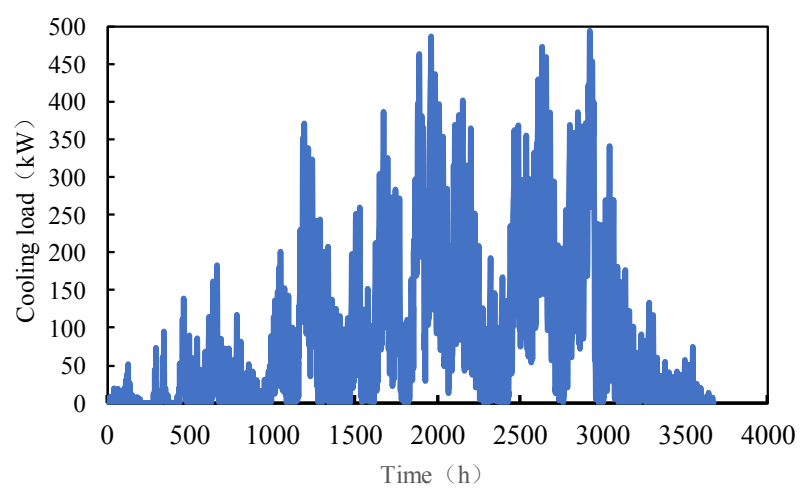

Fig. 4. Annual hourly cooling load of the metro station

The metro station is simulated in TRNSYS 18. The hourly total cooling load, building envelope load, occupant load, equipment load and fresh air load are collected as samples for the NILM method. The annual hourly cooling load is shown in Fig. 4. The time starts at 1:00 am on May 1 and ends on 12:00pm on September 30. The peak cooling load of the metro station is $495.13 \mathrm{~kW}$.

\section{Result analysis and discussion}

In total 1965 samples are obtained from the simulation. All the data were divided into three days. Two days of the three days were taken as the training set, and the rest was taken as the test set. Then the outliers of test set and training set are removed. At last, 1183 samples are used for training and 782 are used for testing. Results are provided as follows.

\subsection{Results based on Fourier transform}

In this section, the results of the ANN model based on the Fourier transform from the total cooling load are analyzed (Approach 1). The total daily cooling load is transferred into the 5th order Fourier series, and then 11 Fourier coefficients are obtained. The coefficients and time are inputted as the feature of disaggregation

The training and testing results are shown in Table 1. The RMSEs are calculated separately for each component load. It can be seen that the all RMSEs for training are less than 7.4, which shows that the ANN can realize the load 
disaggregation accurately. The RMSE for equipment load is the lowest. That is because the cooling load results from the equipment is almost constant in the metro station. The lighting does not need automatic control due to the underground conditions. The occupants load can be disaggregated accurately with a RMSE of 2.8 .

Table 1. Training and testing results of the ANN model based on Approach 1

\begin{tabular}{|c|c|c|}
\hline \multirow{2}{*}{ Item } & \multicolumn{2}{|c|}{ RMSE } \\
\cline { 2 - 3 } & Training & Testing \\
\hline Occupant load & 2.80 & 4.05 \\
\hline Fresh air load & 7.39 & 79.85 \\
\hline $\begin{array}{c}\text { Building } \\
\text { envelope load }\end{array}$ & 3.73 & 17.48 \\
\hline Equipment & 0 & 0 \\
\hline
\end{tabular}

The results for testing samples are shown in Table 1 and Fig.5 8. It can be seen that the results are not as good as that for training samples and the RMSEs are higher. It can be seen that the occupant load (Fig. 4) and equipment load (Fig. 5) from the ANN model matches well with that from the samples. The occupant load changes daily and the equipment load almost keeps constant. For the fresh air load (Fig. 6), the difference between the predicted and the real data is a little large. For some day, the loads match well while the left is not acceptable. For the building envelope load, the data in some periods are very good. The trend is almost consistent and most differences are less than $20 \mathrm{~kW}$. The data from the ANN model is lower than the samples.

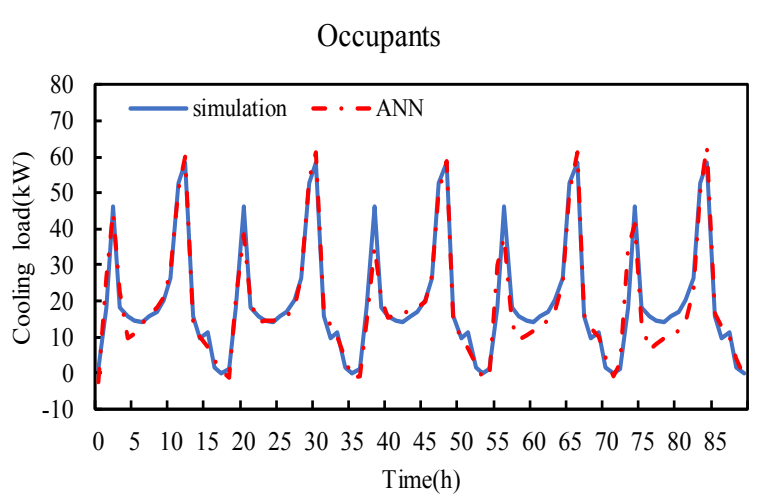

Fig. 5. Testing results for occupant load using Approach 1

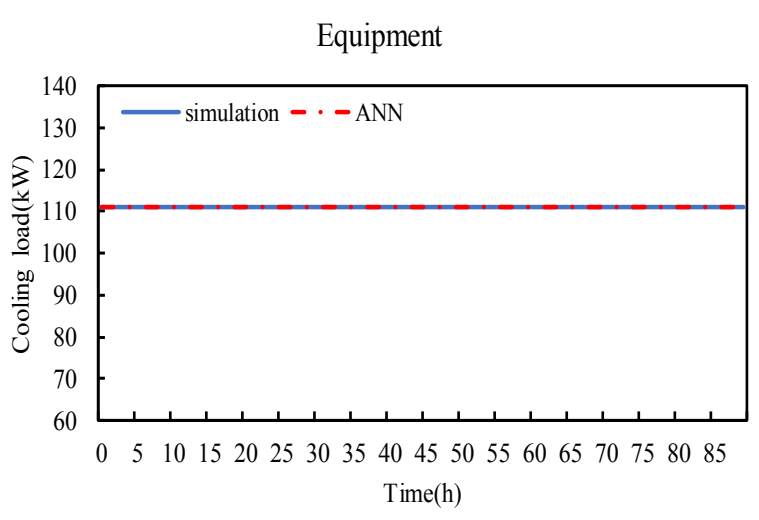

Fig. 6. Testing results for equipment load using Approach 1

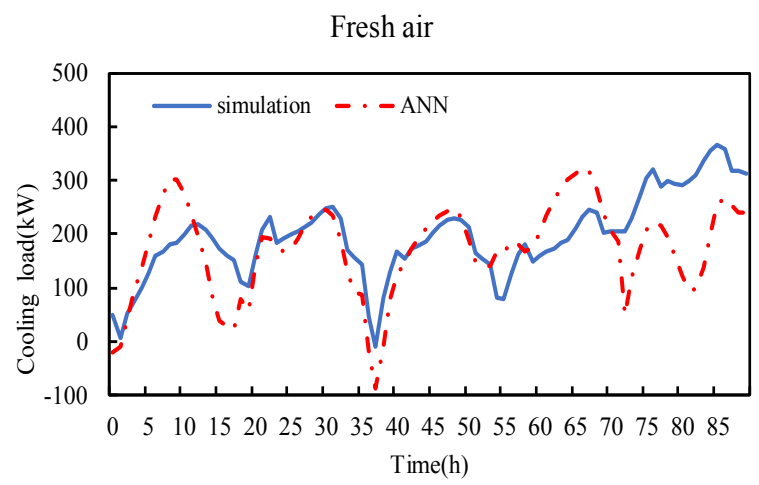

Fig. 7. Testing results for fresh air load using Approach 1

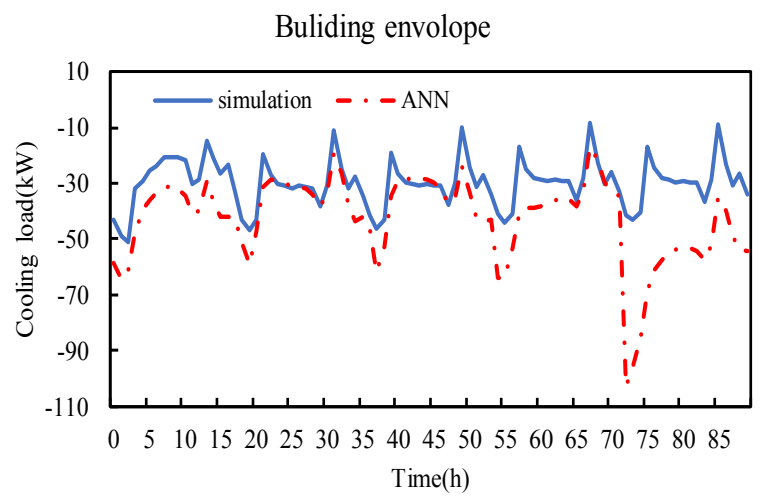

Fig. 8. Testing results for building envelope load using Approach 1

\subsection{Results based on direct total load and outdoor conditions}

In this section, the results of the ANN model based on the load and outdoor weather are presented. The inputs are the total cooling load, the outdoor dry-bulb temperature, the relative humidity and time (Approach 2).

Table 2. Training and testing results of the ANN model based on the Approach 2 


\begin{tabular}{|c|c|c|}
\hline \multirow{2}{*}{ Item } & \multicolumn{2}{|c|}{ RMSE } \\
\cline { 2 - 3 } & Training & Testing \\
\hline Occupant load & 2.09 & 3.42 \\
\hline Fresh air load & 7.25 & 11.20 \\
\hline $\begin{array}{c}\text { Building } \\
\text { envelope load }\end{array}$ & 7.12 & 11.16 \\
\hline Equipment & 0 & 0 \\
\hline
\end{tabular}

The training and testing results are shown in Table 2. The RMSEs are calculated separately for each component load. It can be seen that the four RMSEs for training are less than 7.3, which shows that the ANN can realize the load disaggregation accurately. The RMSE for equipment and the occupant is the lowest. It means that the equipment load and occupant load can be disaggregated from the total cooling with the highest accuracy.

The RMSEs for testing samples are higher than that for training samples. The disaggregation results from each type of load are shown in Table 2 and Fig. 9 12. It can be seen that the occupant load and equipment load from the ANN model matches well with that from the samples. For the fresh air load, the difference between the predicted and the real data is a little large. However, most of the relative difference is less than $15 \%$, which is still acceptable. For the building envelope load, the data in some periods are very good and abnormal points exist.

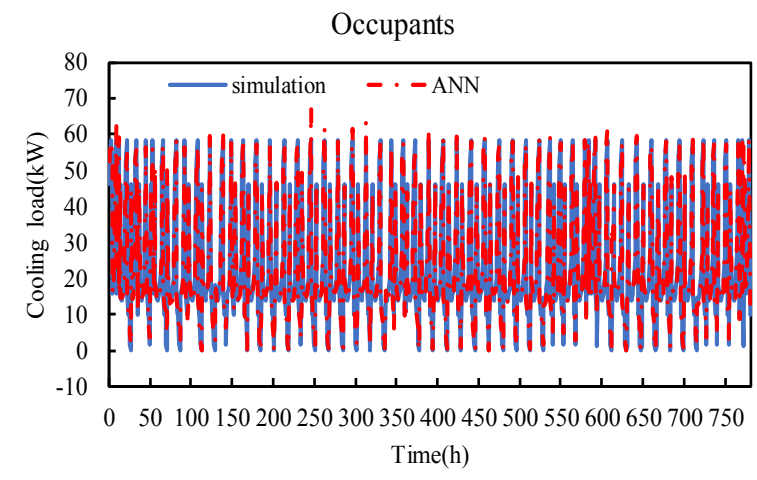

Fig. 9. Testing results for occupant load using Approach 2

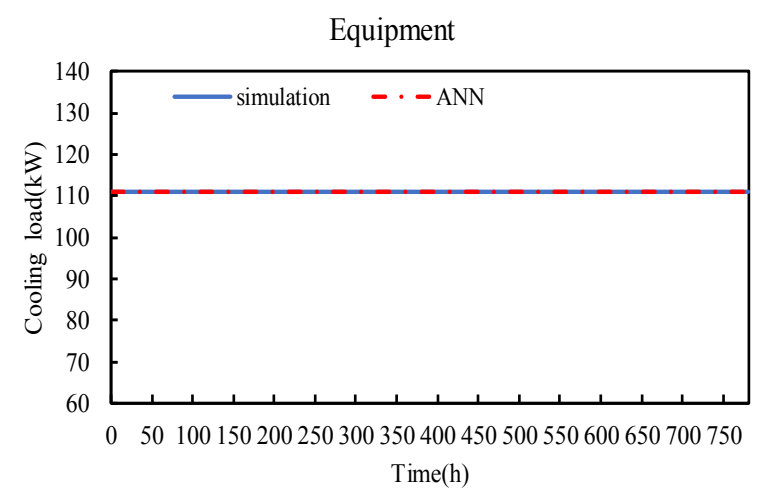

Fig. 10. Testing results for equipment load using Approach 2

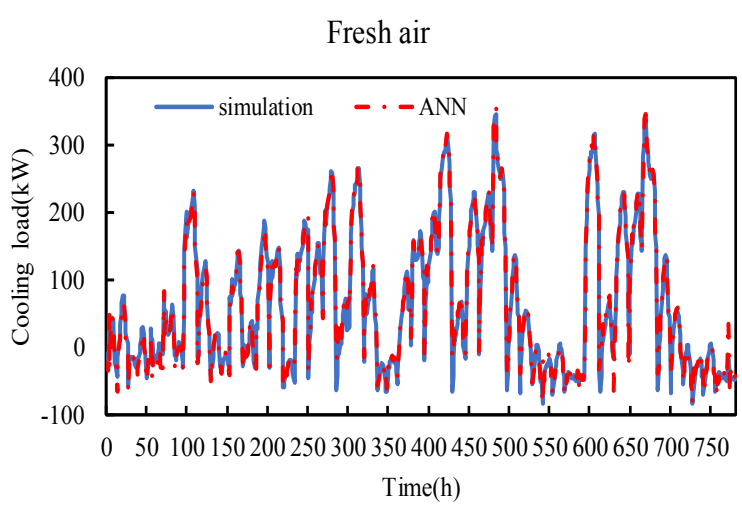

Fig. 11. Testing results for fresh air load using Approach 2

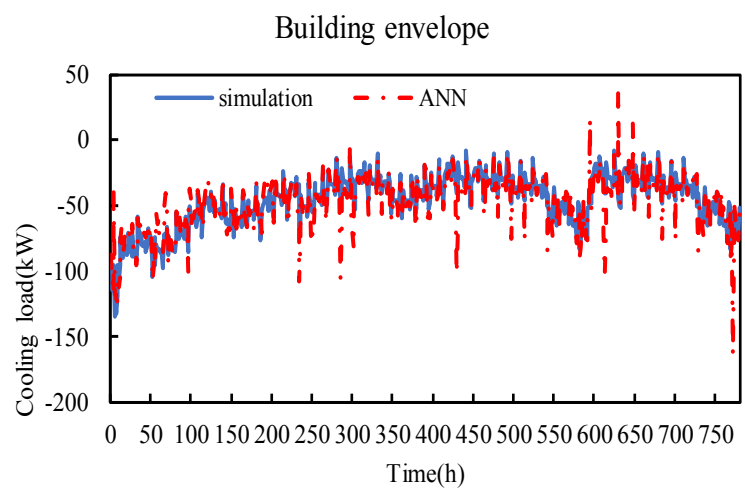

Fig. 12Testing results for building envelope load using Approach 2

\subsection{Discussions}

By comparing the results from 4.1 and 4.2 , it can be found that the approach that uses the cooling load, dry-bulb temperature, relative humidity and time as inputs is better than that based on the Fourier series. Although the RMSEs for training samples are similar, the RMSEs for each type of load are much lower for the testing samples. The RMSE for fresh air load is decreased significantly (from 79.85 to 11.2) and the disaggregation results are acceptable. It means such approach has a stronger disaggregation ability and should be preferred for the cooling load NILM. However, when using the coefficient of the Fourier transform and time as inputs, the parameters to be monitored are only the total load. While using outdoor parameters and total load as input parameters, it is also necessary to monitor the outdoor temperature and humidity.

\section{Conclusions}

A NILM method for metro station is proposed in this study to realize cooling load disaggregation. The cooling loads are disaggregated into four types of loads: occupant load, building envelope load, fresh air load and equipment load. ANN is used to realize the load disaggregation. Two 
approaches are implemented. According to a case study in a metro station, the following conclusions can be obtained:

- The approach based on the Fourier transform of the cooling loads can realize the load disaggregation. The RMSE of the testing results is between 0 and 79.85. The accuracy for occupant load and equipment load is very high. However, for the fresh air and building envelope load, the difference is acceptable in most of cases. During some time, the difference can be very large.

- The approach that uses the cooling load, dry-bulb temperature, relative humidity and time as inputs can realize the load disaggregation. The RMSE of the testing results is between 0 and 11.2. Similar to the former method, the accuracy for occupant load and equipment load is very high.

- The later approach can have a higher accuracy compared with the former one, which is recommended. The results for each type of the load are acceptable.

- When sensors are very limited to collect information, the first approach is preferred because only the cooling load is required as inputs.

The method proposed in this study can realize the NILM of cooling load. However, the accuracy for the fresh air load and building envelope load still needs improvement. Future work will be conducted to improve the load disaggregation by optimizing the models such as the structure of the ANN model, the selection of samples, etc.

\section{References}

[1] Zoha A, Gluhak A, Imran MA, Rajasegarar S. NonIntrusive Load Monitoring Approaches for Disaggregated Energy Sensing: A Survey. Sensors. 2012;12(12):16838.

[2] Hart GW. Nonintrusive appliance load monitoring. Proceedings of the IEEE. 1992;80(12):1870-91.

[3] Zeifman M, Roth K. Nonintrusive Appliance Load Monitoring: Review and Outlook. IEEE Transactions on Consumer Electronics. 2011;57(1):76-84.

[4] Advanced metering initiatives and residential feedback programs: a meta-review for household electricity saving opportunities.

[5] Basu K, Debusschere V, Bacha S, Hably A, Van Delft D, Jan Dirven G. A generic data driven approach for low sampling load disaggregation. Sustainable Energy, Grids and Networks. 2017;9:118-27.

[6] Cominola A, Giuliani M, Piga D, Castelletti A, Rizzoli AE. A Hybrid Signature-based Iterative Disaggregation algorithm for Non-Intrusive Load Monitoring. Applied Energy. 2017;185:331-44.

[7] Wang Y, Li X. Unorganized ventilation in subway stations with Platform Screen Doors. Building and Environment. 2017;125:556-64.

[8] Zhang G, Eddy Patuwo B, Y. Hu M. Forecasting with artificial neural networks:: The state of the art. International Journal of Forecasting. 1998;14(1):35-62. 